\title{
COVED: A Hardware Accelerated Soft Computing Enabled Intelligent Value Chain Based Diagnostic Automation for nCOVID- 19 Estimation and Identification
}

\author{
Swarnava Biswas ${ }^{1}$, Debajit Sen ${ }^{2}$, Dinesh Bhatia ${ }^{3}$ and Moumita Mukherjee ${ }^{4, *}$ \\ ${ }^{1}$ The Neotia University, Kolkata, West Bengal, India \\ ${ }^{2}$ Robert Bosch Engineering and Business Solutions, Bangalore, Karnataka, India \\ ${ }^{3}$ Department of Biomedical Engineering, North Eastern Hill University (NEHU), Shillong, Meghalaya, India \\ ${ }^{4}$ Department of Physics, School of Basic and Applied Sciences, Adamas University, Kolkata, West Bengal, \\ India
}

\begin{abstract}
Purpose: COVID-19, a global pandemic, first appeared in the city of Wuhan, China, and has since spread differently across geographical borders, classes, and genders from various age groups, sometimes mutating its DNA strands in the process. The sheer magnitude of the pandemic's spread is putting a strain on hospitals and medical facilities. The need of the hour is to deploy loT devices and robots to monitor patients' body vitals as well as their other pathological data to further control the spread. There has not been a more compelling need to use digital advances to remotely provide quality healthcare via computing devices and $\mathrm{Al}$-powered medical aids.

Method: This research developed a deployable Internet of Things (IoT) based infrastructure for the early and simple detection and isolation of suspected coronavirus patients, which was accomplished via the use of ensemble deep transfer learning. The proposed Internet of Things framework combines 4 different deep learning models: DenseNet201, VGG16, InceptionResNetV2, and ResNet152V2. Utilizing the deep ensemble model, the medical modalities are used to obtain chest high-resolution computed tomography (HRCT) images and diagnose the infection.

Results: Over the HRCT image dataset, the developed deep ensemble model is collated to different state-of-the-art transfer learning (TL) models. The comparative investigation demonstrated that the suggested approach can aid radiologists inefficiently and swiftly diagnosing probable coronavirus patients.

Conclusion: For the first time, our group has developed an Al-enabled Decision Support System to automate the entire process flow from estimation to detection of COVID-19 subjects as part of an Intelligent Value Chain algorithm. The screening is expected to eliminate the false negatives and asymptomatic ones out of the equation and hence the affected individuals could be identified in a total process time of 15 minutes to 1 hour. A Complete Deployable System with AI Influenced Prediction is described here for the first time. Not only did the authors suggest a Multiple Hypothesis based Decision Fusion Algorithm for forecasting the outcome, but they also did the predictive analytics. For simple confined isolation or hospitalization, this complete Predictive System was encased within an loT ecosystem.
\end{abstract}

Keywords: Artificial Intelligence, Internet of Things, Deep Learning, Machine Learning, COVID-19 Detection, HRCT, Pathology Data, Stage Classification COVID-19, Raspberry Pi, Intel Movidius $^{\mathrm{TM}}$, Neural Compute Stick.

\section{INTRODUCTION}

Smart cities, industry, home automation, and medical have all embraced loT devices in recent years [1]. Using data analytics, [2] which include direct and continuous engagement with people and natural language processing that analyses massive quantities of people's health data [3], the priority has changed away from cure and toward prevention. Due to the anticipated presence of coronavirus infection in the future, diagnostics from a remote location will be necessary due to a shortage of point-of-care facilities [4]. The Internet of Things (IOT) is changing healthcare based on the cost to value, which increases existing medical centers that are restricted by facilities [5]. The

*Address correspondence to this author at the Department of Physics, School of Basic and Applied Sciences, Adamas University, Kolkata, West Bengal, India; E-mail: moumita.mukherjee@adamasuniversity.ac.in world's healthcare system is currently swamped by the COVID-19 pandemic. This is the reason why the research group thought of proposing a solution for an loT-enabled healthcare to make prevention \& control the spread of coronavirus pandemics as part of the modern health informatics system [6].

The motivation for such a work can be explained by the fact that the world was found wanting a solution during the viral outbreak's peak stages in the majority of countries [7]. The requirement for additional tests to accurately identify and diagnose was a pressing need that some countries failed to meet. One possible reason is the real-time polymerase chain reaction (RTPCR) test and the associated costs on both the facility and testing side. As a result, some of the local diagnostic centers were unable to adopt the same for mass testing. The other disadvantage of this method 
was the lengthy turnaround time, more than 48 hours to be precise [8]. Thus, our group has attempted to propose a reliable estimation and screening process that will prepare diagnostic centers for future spikes and proper identification using the diagnostic center's available facilities.

Additionally, the authors propose a two-step screening process for accurate disease diagnosis. The total time required for screening is estimated to be between 15 and 1 hour. The first step is to evaluate the patient using body sensors in conjunction with symptomatic analysis. The second step entails the evaluation of high-resolution computed tomography (HRCT) images, as well as the analysis of pathological data obtained from routine blood examinations and body sensor vitals. Thus, the novelty of this paper is best described by the multi-fold methodology described above for the binary classification of the patients.

\section{LITERATURE SURVEY}

The benefits of the medical Internet of Things (IoT) have played a major role in the effort to prevent the COVID- 19 pandemic. Deep learning (DL) models in combination with loT may be created to free up medical staff and doctors to handle additional patients. Based on studies done with coronaviruses, it has been shown that several research papers have shown that many suspected instances of infection were discovered employing DL models which are used to identify coronavirus using X-ray, CT scan, and ultrasound as input [9]. Many researchers have employed Convolutional Neural Networks (CNNs) for image categorization, most of which create distinct neural network topologies. Deep convolutional neural networks are a sophisticated deep learning architecture used in many machine learning tasks. According to [10], CNN's can train weights from scratch using a big dataset, fine-tune weights from a pre-trained CNN using smaller datasets, and pre-train $\mathrm{CNN}$ as a feature extractor. Deep learning is commonly used on chest radiography pictures to detect COVID-infected patients, with promising results in terms of accuracy. [11] Presents a deep convolutional neural network that can detect coronavirus illness from chest $X$-ray pictures. The proposed CNN uses pre-trained transfer models (ResNet50, InceptionV3, and Inception-ResNetV2) to predict X-ray images with high accuracy. The photos are normal and COVID-19. To offset the lack of data and training time, the ImageNet dataset is used to perform transfer learning. The ResNet50 model outperformed the other models among both training and testing. A new Framework based on transfer learning and class decomposition was described by Abbas et al. [12]. The suggested architecture, DeTraC, includes three phases. First, a pre-trained ImageNet $\mathrm{CNN}$ extracts local features. Final categorization of images using error-correction criteria applied to a softmax layer is performed using the stochastic gradient descent training method. The ResNet18 pretrained ImageNet network achieved $95.12 \%$ accuracy on CXR pictures. In the next cited article, the authors have developed a system based on IoT modalities for early-stage detection of affected individuals. Previously ResNet101 in combination with Faster Region CNN was used to identify infected cases that were suspected positive with $98 \%$ accuracy [13]. Multiple ConvNet models such as CheXNet, SqueezeNet, ResNet18, ResNet101, VGG19, DenseNet201, Inceptionv3 and, MobileNetv2 were used in this research structure. This methodology was tested on the coronavirus infected cohort of 423 cases of COVID virus infection, the 1485 cases of viral pneumonia, and the 1579 patients who presented with non-diagnostic chest X-ray (CXR) images. COVID- 19 was distinguished from other infections using a 3D convolutional neural network (3DCNN) [14]. A strategy for a fully automated screen for COVID-19 was developed based on attention-based deep 3D multiple instance learning (AD3D-MIL) [15]. The AD3D-MIL learned using the Bernoulli distribution of labels, making the method highly efficient.

A proposed M3 Lung-sys CT screening system is a multi-task multi-slice deep learning system that aids in the finding of people infected with coronaviruses [16]. The framework was inspired by the current advancement of DL models for the detection of coronavirus disease with automation. To find out if the patients have COVID-19 (+) or healthy conditions, the proposed model based on ensemble learning (EL) will be helpful to both the doctors and healthcare staff. The automated diagnosis of suspected COVID-19 cases is handled by a deep ensemble learning model and loTbased architecture that has been built specifically for this purpose. This ensemble model incorporates InceptionResNetV2, ResNet152V2, VGG16, and DenseNet201, and works such that it ensembles them together. Deep ensemble learning is used to identify an infection with medical sensors that monitor chest X-ray methodologies. This dataset was used for experimental purposes and had two classes (COVID-19 (+), or healthy). In comparison to existing models, the proposed model will prove to be effective for the 
detection of coronavirus suspected patients with greater efficiency and expediency. AD3D-MIL was given 460 CT images for training and testing. Zhang et al. [17] developed a new deep anomaly detection model for COVID- 19 screening using CXR images. The suggested model includes a backbone network, classification heads, and anomaly detection heads. The backbone network extracts high-level visual features for the categorization and anomaly detection head. The classification head uses a new classification convolutional layer with a hidden layer of 100 neurons, an output layer of one neuron, and the "sigmoid" activation function. In contrast to classification, anomaly detection provides scalar anomaly scores, which in turn identify anomaly images (COVID-19 cases). The proposed model reduced false positives. The results showed 96.00 percent sensitivity and 70.65 percent specificity. [18] presents COVID-Net, a deep convolutional neural network that can recognize COVID-19 cases from CXR images. The network architecture uses a lightweight residual projectionexpansion-projection- extension (PEPX) design pattern and consists of two stages: human-machine collaborative design and machine-driven design exploration. For decision validation, an explainabilitydriven audit is undertaken. COVID-19 instances had great sensitivity $(87.1 \%)$ and precision $(96.4 \%)$.

In [19] the authors looked into using technology to help diagnose patients afflicted with the virus. Pretrained convolutional neural networks were tested for their ability to detect infection in chest X-ray pictures. A dataset was built using publicly available $X$-ray scans of COVID-19 patients, common bacterial pneumonia patients, and healthy people. To compensate for the small sample size, we used transfer learning, which transfers knowledge from pre-trained models to the new model. The experimental findings show that the best two models can classify with $95 \%$ accuracy.

In the next article, a dual-sampling technique, as well as online attention refinement was used, in the development of DCNN. This network was employed to locate the infection locations and redistribute pneumonia-infected locations evenly. The DCNN was used on 2,757 CT scan images of 2,057 patients, according to our tests. As a result, it is reasonable to assume that the affected region's accuracy is still not yet up to the mark. DL-CRC, chest radio classification based on DL, was proposed for identifying persons with confirmed coronavirus infection using chest X-ray [20]. To create artificial coronavirus infected X-ray images, the DL-CRC used data augmentation along with a generative adversarial network. Multiple CXR datasets were evaluated using DL-CRC. Another study [21] uses a CNN framework to detect COVID-19 in pneumonia cases. The COVID-ResNet framework fine-tunes a pretrained ResNet-50 framework in three steps to improve effectiveness and decrease training time. The gradual scaling of input images $(28 \times 128 \times 3,224 \times 224 \times 3$, $229 \times 229 \times 3$ ) and fine-tuning of the network at each stage improves generalization and overall performance (96.23 percent accuracy). COVIDX-Net is a network consisting of seven deep learning image classifiers designed to classify COVID-19 illness from CXR pictures. The results showed that the DenseNet201 and VGG19 classification methods had the best performance with $90 \%$ accuracy. With Monte Carlo Drop weights Bayesian convolutional neural networks, the authors researched how to assess uncertainty in deep learning to better human-machine decisions. A Bayesian Deep Learning classifier was trained using COVID-19 X-Ray pictures to quantify model uncertainty. As a result of this substantial association, incorrect predictions can be identified.

The generative adversarial network (GAN) was developed as an auxiliary classifier model that generates synthetic CXR images. CovidGAN, the developed framework, was called into existence and used to differentiate other viral pneumonia with coronavirus infections [22]. CovidGAN was put to the test by subjecting $192 \mathrm{CXR}$ images to analysis. It is unknown whether any type of cross-validation has been performed on the architecture CovidGAN. In the next article, for developing an automatic technique to classify images VGG19 was used. Image quality was improved by using pre-processing techniques to reduce the bias of the samples. Classification accuracy can, however, be improved by data fusion approaches. a new transfer learning (TL) strategy for the categorization of coronavirus suspected patients was introduced using a ConvNet. [14].

An ensemble deep transfer learning model was designed by Gianchandani et al. [23] to identify suspected patients who are members of the COVID-19 infected population. To test COVID-19-infected patients, a deep neural network-based screening model was implemented by Singh et al. [24]. Again densely attached ConvNets were used by Singh et al. [25] to classify infected patients. On the other hand, the models show increased efficiencies, however additional advancement can also be made utilizing ensemble models. Finally, Apostolopoulos et al. [26] tested five pre-trained CNN networks for COVID-19 identification 
from CXR. VGG19 and MobileNetv2 had the highest accuracy, 93.48 and 92.85 percent, respectively.

Following an analysis of current literature, it was determined that existing approaches are indeed afflicted by the overfitting problem [27, 28]. Ensemble models, as opposed to individual models, make use of a variety of learning approaches to achieve superior classification performance. When deep learning models exhibit a higher variability, their ensemble delivers statistically meaningful results [29]. To enhance predictions and reduce the variance and bias, different types of deep learning models are combined into a single categorization model called an ensemble.

RT-PCR is an excellent tool for more precisely identifying normal categories. Additionally, the approach reliably identifies COVID-positive people, with classification accuracy ranging from 65 to 95 percent [30]. While the standard RTPCR-based approach accurately predicts normal categories, COVID patients' risk is unnoticed. While the diagnostic has a high rate of correctly identifying COVID patients from a radiological viewpoint, this is not true for the typical category of individuals. The majority of studies have concluded at the level of detection, i.e. COVID or healthy patient. However, the authors have taken this effort a step further by integrating the entire value chain into an IoT ecosystem to leverage the combined powers of $\mathrm{Al}$ and loT to detect and isolate coronavirusinfected individuals. To emphasize, a further developed Al model anticipates the various groups of coronavirusinfected patients, while the loT system supports pointof-care institutions in separating and prescribing the necessity of hospitalization for COVID patients.

In a word, this entire algorithmic approach may be used for both predictive and prescriptive analytics to complete the diagnostic pipeline from the physician's perspective. Indeed, we enhanced the overall system's accuracy and prediction by combining a supervised learning strategy with a multi-hypothesis-based decision fusion technique.

\section{METHODOLOGY}

Our proposed mathematical model revolves around radiography image viz-a-viz High-Resolution Computed Tomography (HRCT) scan as the pivot point around which the prediction for Detailed Detection and diagnosis of COVID-19 is organized. In this way, it could yield crucial data in the process of screening. Additionally, the technique would be utilized to aid in the use of the conventional PCR-based method for isolating the individuals who have contracted. To detect coronavirus patients, we'll be using a DL model in combination with a classical Machine Learning (ML) model.

The proposed framework is used for analyzing the binary class HRCT images. The suggested ensemble framework is compared to present state-of-the-art deep transfer learning models. The experiments are run on MATLAB 2020b software with a Core i7 $3.80 \mathrm{GHz}$ processor, 32 GB RAM, and a $15 \mathrm{M}$ cache. To overcome the overfitting problem, this study employs 20 -fold cross-validation. For training purposes, $70 \%$ of the total dataset is considered.

\subsection{Dataset}

The authors used an open-source resource that contains over 1,000 CT scans of lung infections of coronavirus patients taken by board-certified radiologists for their investigation. CT scans were performed at two large hospitals in Mashhad, Iran, between March 2020 and January 2021. Viral loads caused due to COVID were authorized with corresponding investigations that comprised reverse transcription-polymerase chain reaction (RT- PCR) and supporting signs and symptoms. All photographs are 16-bit grayscale with a resolution of $512 \times 512$ pixels and those are recorded in DICOM format. The privacy of patients is safeguarded by the removal of medical data from images.[31]. After that, all patient photographs are compressed and archived in the RAR format. Additionally, data is enhanced through random rotation, cropping, and blurring.

\subsection{Body Vitals Data-Based Machine Learning Model Development}

First, an array of sensor readings, such as temperature, saturation percentage of oxygen $\left(\mathrm{SpO}_{2}\right)$, and others, are taken from various parts of the body. A machine learning technique called linear discriminant analysis is used to predict the patient's health outcome. For better classification, our Deep Network Architecture (DNA) performs on the HRCT scan-based analysis of the data.

Visitors to a commercial, housing complex, or even a hospital are more likely to be screened with a thermal gun and given a set of questionnaires these days than previously. Identifying suspicious cases at the entry point can be helpful during the process. Our goal is to remove the human factor and human error from the 
Table 1: Pathological Data-Based Machine Learning Model Development Parameters

\begin{tabular}{|c|c|c|c|c|c|}
\hline \multicolumn{2}{|c|}{ MODEL: OPTIMIZABLE ENSEMBLE } & \multicolumn{2}{|c|}{ OPTIMIZED HYPERPARAMETERS } & \multirow{2}{*}{\multicolumn{2}{|c|}{$\begin{array}{l}\text { OPTIMIZER OPTIONS } \\
\text { Bayesian Optimization }\end{array}$}} \\
\hline Learner Type & Decision Tree & Ensemble Method & Bag & & \\
\hline Accuracy & $98.82 \%$ & $\begin{array}{l}\text { Maximum No of } \\
\text { Splits }\end{array}$ & 556 & Training Time & $300 \mathrm{~S}$ \\
\hline $\begin{array}{c}\text { Total } \\
\text { Misclassification Cost }\end{array}$ & 104 & No of Learners & 58 & Iterations & 30 \\
\hline Prediction Speed & $\begin{array}{l}\sim 790 \\
\text { observations/sec }\end{array}$ & $\begin{array}{l}\text { No of Predictor } \\
\text { Samples }\end{array}$ & 4 & Training Time & TRUE \\
\hline Training Time & $312.59 \mathrm{sec}$ & & & & \\
\hline
\end{tabular}

equation, thus reducing the number of mistakes. To classify the COVID $(+)$ or the healthy individual according to temperature and oxygen saturation percentage, we've used an Al-based classification tool that we have trained on a huge data set. Information for correct classification of COVID individuals was extracted from a dataset containing age, gender, temperature, and $\mathrm{SpO}_{2}$ as parameters and trained in MATLAB using the Classification Learner $A p p^{\circledR}$, with the model with the highest accuracy returned as the solution. The details of the training model are listed above in Table 1. Bag [32], Ada Boost [33], and RUS Boost [34] were tested individually using the ensemble method. We conducted a test in which we changed the number of learners from 10 to 500 , varied the learning rate from 0.001 to 1 , set the number of splits at 560 , and tried out different numbers of predictors. To avoid confusion, the Cost Matrix for Misclassification was set to Default and PCA was disabled. This model returns as specified above.

\subsection{Pathological Data-Based Machine Learning Model Development}

In the second step, another secondary hypothesis is used to generate a new dataset that uses another machine-learning algorithm to analyze the complete blood count (CBC). The dataset included age, gender, Leukocyte, Lymphocyte, and Neutrophil count as training parameters and used the Classification Learner App in MATLAB to build a model that could identify the COVID individuals with the highest accuracy. Patients with coronavirus load commonly find a decrease in lymphocyte count and increase in CRP and highsensitivity C-reactive protein (HSCRP) level [35-37]. Table 2 contains training model details, along with visualizations for evaluating performance. A separate study was conducted to test the Optimizable Tree method for maximum deviance reduction [38], Towing Rule [39], and Gini's Diversity Index [40]. It was tried to examine how many splits there are from 1 to 560 . To keep the cost of misclassification low, the Cost Matrix for Misclassification was kept as the default and PCA was disabled. The Final Model received was returned in its original state as described above.

\subsection{Ensemble Learning Model for HRCT Scan Based Detection}

By a more informative imaging technique, the CT Scan based detection technique would allow the Al model to draw more distinctive inferences. When a detailed diagnosis is required, the technique is a highly popular one. In this remarkably similar manner, our confirmatory diagnostic screening phase III would be for individuals who have a COVID-19 found in their screening phase II. The proposed COVID-19 ensemble model is illustrated in Figure 1 below.

Table 2: Body Vitals Data-Based Machine Learning Model Parameters

\begin{tabular}{|c|c|c|c|c|c|}
\hline \multicolumn{2}{|c|}{ MODEL: OPTIMIZABLE TREE } & \multicolumn{2}{|c|}{ OPTIMIZED HYPERPARAMETERS } & \multirow{2}{*}{\multicolumn{2}{|c|}{$\begin{array}{l}\text { OPTIMIZER OPTIONS } \\
\text { Bayesian Optimization }\end{array}$}} \\
\hline Surrogate decision splits & OFF & Maximum No of Splits & 73 & & \\
\hline Accuracy & $98.78 \%$ & \multirow[t]{2}{*}{ Split } & \multirow{2}{*}{$\begin{array}{l}\text { Max deviance } \\
\text { reduction }\end{array}$} & Training Time & $300 \mathrm{~S}$ \\
\hline Total Misclassification Cost & 101 & & & Iterations & 30 \\
\hline Prediction Speed & $\begin{array}{c}\sim 17000 \\
\text { observations/sec }\end{array}$ & & & Training Time & TRUE \\
\hline
\end{tabular}




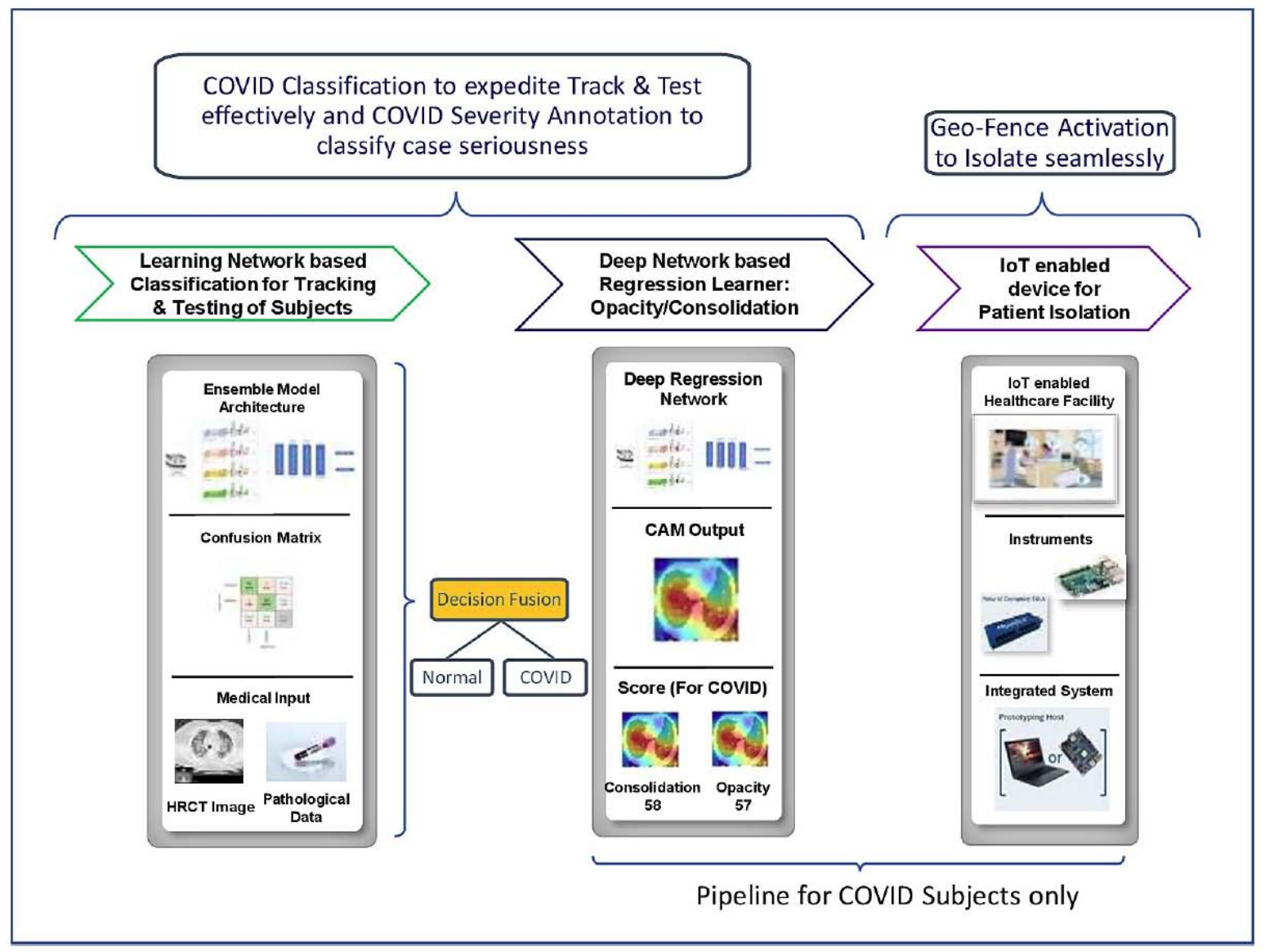

Figure 1: The complete solution pipeline for the deployable architecture.

Transfer learning machine learning models known as ResNet152V2 [14], DenseNet201 [14], VGG16 [41], and InceptionResNetV2 [42] are ensembled in a new model for greater efficiency. The reason for the selection of these models is that these models achieve an excellent accuracy in testing the dataset we've collected, and they have greater diversity when it comes to implementing deep learning models. Using an ensemble of pre-trained models is more efficient than using a single model. Using the ensemble approach has several advantages, including the ability to identify the most important characteristics and enhance the classification accuracy. A depiction of the proposed model for the diagnosis of coronavirus is shown in Figure 2.

The dense layer requires 64 neurons. The features are extracted using many layers of a finely tuned transfer learning model. A softmax activation function is used to deal with binary-class classification problems. Epochs are used, and batch size is set to 10 . Using connected layers, the number of neurons is 64 and dropout at 0.3 and 0.2 respectively to prevent overfitting is utilised during the tuning of attributes. Concepts of early stopping are incorporated into regularization. When calculating a learning rate, 0.001 is typically used. Since we're only dealing with the Binary Class Problem, i.e. COVID \& NORMAL, we've restricted our classification problem to that. To highlight the effectiveness of a combined hypothesis, which is based on the pathological study as described above, and Al-enabled radiography-assisted techniques for COVID detection, this paper also talks about the synergistic effect of the combined hypothesis and Alassisted radiography. To identify "COVID" and "NORMAL" subjects, we are using this model. Additionally, the researchers have tried to group "COVID" based on CT scan images to elucidate different stages.

We can explain the lung condition, as we have mentioned, about the previously described symptoms. Ground Glass Opacity (GGO) and Lung Consolidation can be defined as the measurement metrics to determine the correct stage. In this case, the phrase "hazy increased opacity in lung parenchyma" means there is cloudiness to the lungs, but it is not obscured by the underlying vessels. Early on, the virus invades the alveolar epithelium, and this leads to the leaky alveolar cavity and an inflamed or thickened alveolar 


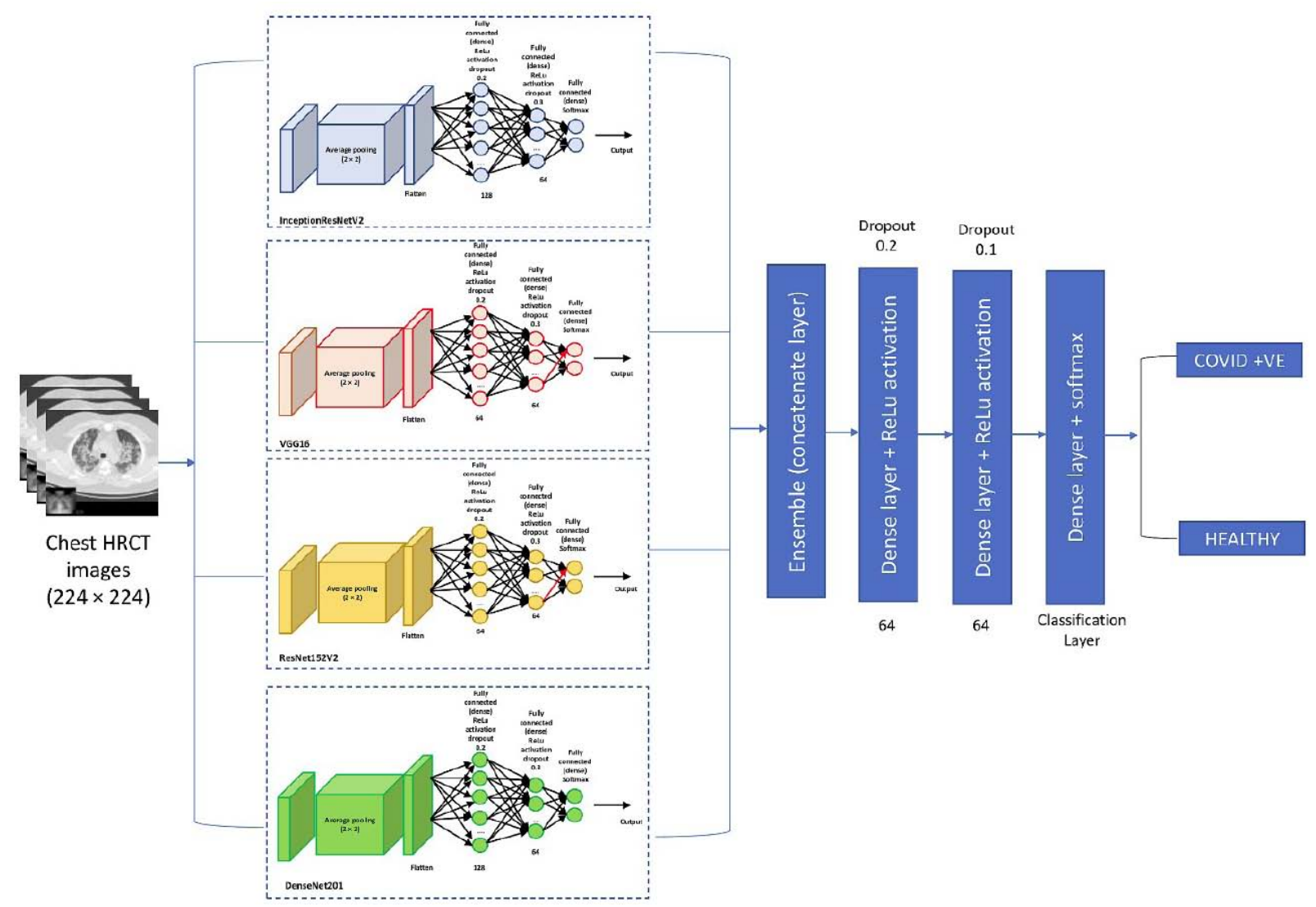

Figure 2: Developed ensemble DL architecture.

wall $[43,44]$. Lung opacification with obscuration of underlying vessels is seen with consolidation. The body reacts to inflammation, causing a large amount of exudation in the alveoli [35]. Most recurrent findings in COVID, as well as bilateral peripheral distribution in particular at the lower zone, are the airspace opacities like ground-glass opacities and consolidations [45, 46]. Parenchymal abnormalities are not found in $3 \%$ of cases [47]. The bilateral bronchopulmonary segment involvement contributes to the severity score [48]. Both lungs were segmented into 20 regions to create 18 segments in total. A score of 0,1 , or 2 can be obtained for each segment depending on whether parenchymal opacification was present, whether it was less than $50 \%$ or greater than $50 \%$, and how much parenchymal opacification was present. COVID-19 with 83.3\% sensitivity and $94 \%$ specificity is defined as severe COVID-19 with an identified optimal score of 19.5.

The developed system uses data fusion to make personalized predictions. To make a final prediction, these different predictions are merged. An Internet of Things-based smart medical system transmits remote physiological and medical data to a monitoring center for analysis and diagnosis [49]. One of the most important aspects of the system is the server-based data fusion mechanism. Data fusion is a powerful tool for combining large amounts of data from various sources. Multi-sensor data fusion combines data from multiple sensors and sources to make inferences that a single sensor or source cannot [50]. On one, the medical loT facilitates the acquisition and integration of medical data, hence promoting health. The medical loT relies on data fusion. It can't avoid huge multisource diversified data integration and sharing issues. The network captures audio, video, data, and text. After clever data processing, personal health promotion reports and tracking interventions are developed [51]. As well as various spatiotemporal elements and data including health, medical, physical results and monitoring, and evaluation. They are scattered among systems with varied data standards and analysis methods. So it must use data fusion to combine medical and health resources. Increasing the value of people's health assets [52]. Many medical Internet of Things investigations has been done abroad [53]. A data fusion tree improves data fusion efficiency and shows the technology's progress.

The first of the two sub-diagnostic measures are based on pathological data and medical vitals of test subjects. In the second screening step, we have 
proposed the idea of combining the conventional radiography-based classification to assist the doctors in identifying any strain. Decision fusion algorithms are important for the multi- hypothesis situation, and can therefore be easily integrated into clinical diagnostic procedures. The concept of multi-hypotheses will be a valuable second opinion to doctors, enabling diagnostic-based approaches. The Dual Pool Hypothesis uses two different algorithms; thus, we propose a new algorithm, "Whoever Scores Most" as a probabilistic metric to correctly identify the subject concerned in decision conflict scenarios. We've finally put the algorithm to work, and it will finally return the class with the highest probability. The equation of our methodology is described below:

C1 represents the output class predicted by the machine learning algorithm for pathological data. S1 represents the probability score for class $\mathrm{C} 1$

C2 represents the output class predicted by the deep learning model for radiographic images S2 represents the probability score for output class $\mathrm{C} 2$
Let say $X$ represents the single output given, two different output from different algorithms. For all output classes,

$X=\operatorname{argmax}\left(S_{1}, S_{2}\right)$

The decision fusion algorithm is described in Figure 3.

\subsection{IoT Enabled Deployability}

Using the concept of the internet of things (IoT), the authors have made this composite system available to diagnostic facilities. People have trusted the Internet of Things for healthcare for quite some time now. As loTbased devices have evolved, so has the concept of telemedicine and remote patient monitoring systems. loT has been used to aid our Al-based patient diagnostic system to increase reliability and performance. The Intel Movidius Neural Compute Stick (NCS) is a new piece of hardware designed to support machine learning models on small, embedded devices. The Intel Movidius ${ }^{\mathrm{TM}}$ USB device can be connected to any device such as laptops, Raspberry $\mathrm{Pi}$, or tablets. We've trained our models to classify COVID-affected

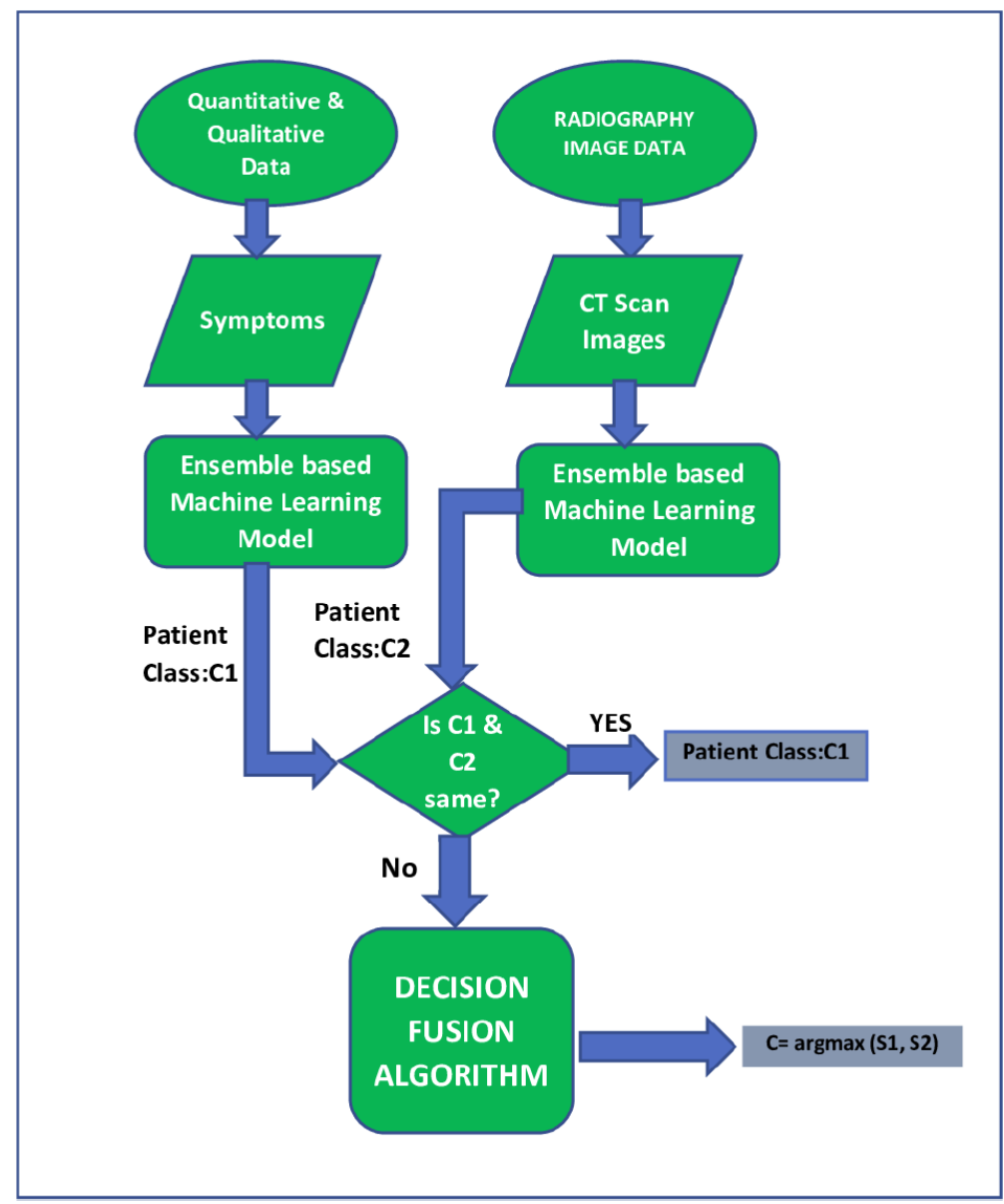

Figure 3: Decision fusion algorithm. 


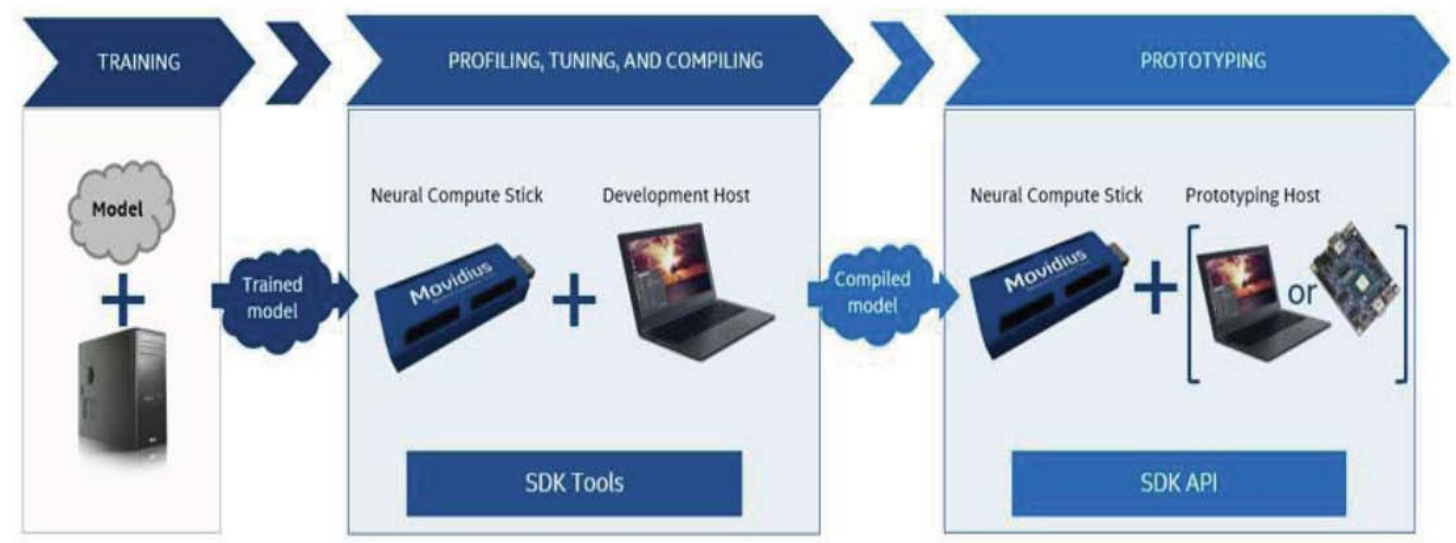

Figure 4: The development process of the NCS is presented in the workflow diagram.

subjects with the Intel Movidius ${ }^{\mathrm{TM}}$ Neural Compute Stick. In the multi-hypothesis scenario, running parallel Ensemble Learning (EL) models is extremely important. We initially developed and trained our models on a powerful host machine (a development computer in our case). To be compatible with the VPU hardware, a previously built model was profiled, tuned, and compiled using an Application Programming Interface (API) that supported the VPU. To validate the new model, the prototype was generated on the same machine, and then the model was checked out. The hardware is physically connected to the machine and was accessed using the APIs available. The development process using NCS is explained in Figure 4.

The philosophy of the software architecture to be used for deployment is described in Figure $\mathbf{5}$.

The steps followed for inference on the edge are explained in Figure 6.

To draw inference from deployed models, the following six basic steps are followed:

- $\quad$ For the server to start up, the computer must be configured to use the localhost as the server

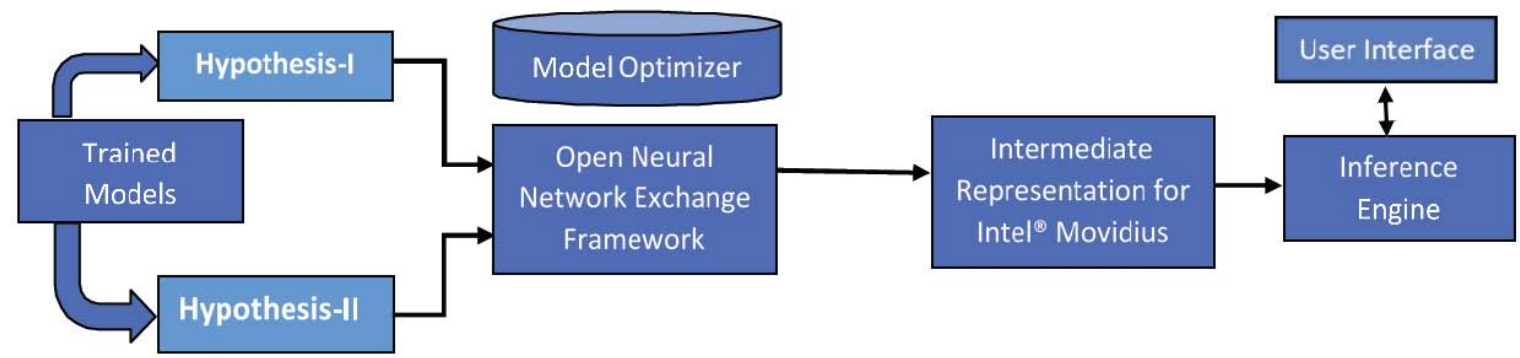

Figure 5: The philosophy of the AI Software Architecture to be used for deployment.

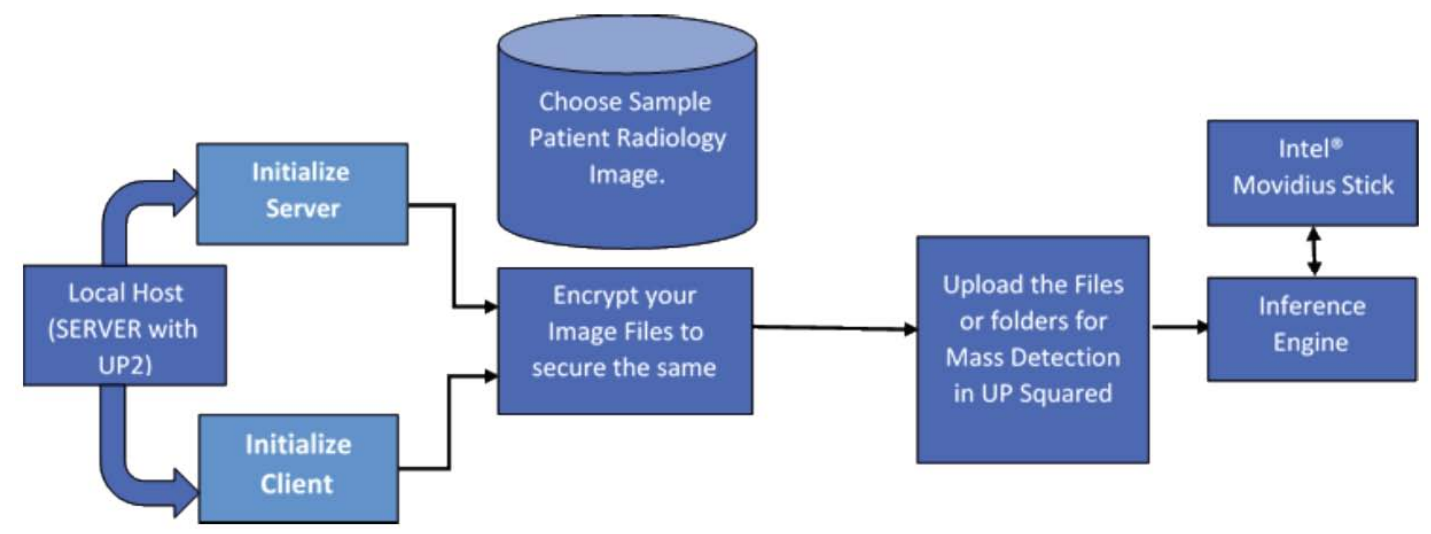

Figure 6: The setting up the server to test a COVID-19 Positive or Normal sample. 
Table 3: Confusion Matrix Table for Different Machine Learning Models

\begin{tabular}{|c|c|c|c|}
\hline \multicolumn{2}{|c|}{ The pathological data-based machine learning model } & \multicolumn{2}{c|}{ Body vitals data-based machine learning model } \\
\hline Evaluation Parameter & Score & Evaluation Parameter & $98.19 \%$ \\
\hline \hline Sensitivity & $98.71 \%$ & Sensitivity & $99.097 \%$ \\
\hline $\begin{array}{c}\text { Specificity } \\
\begin{array}{c}\text { The area under the curve (AUC) of } \\
\text { ROC }\end{array}\end{array}$ & $98.87 \%$ & Specificity & 0.94 \\
\hline Misclassification Error & 0.92 & Misclassification Error & 0.18 \\
\hline
\end{tabular}

address. It's the "UP Squared Device" in our case. The NCS is connected to a piece of hardware known as 'UP Squared Device' developed by Intel.

- To ensure that only those with permission to access the data could access it, a set of encryptions was applied.

- $\quad$ Each radiography image was uploaded to the UP Squared where it was used as part of the server.

- A set of Radiography Images can be inferred with the models running on the Intel $\AA$ Movidius $^{\mathrm{TM}}$ stick.

- $\quad$ No matter what type of machine was used, using the Intel Movidius device on the 'UP Squared device' did not change the classification accuracy compared to development machines, such as a Windows 10 Home with an Intel Core i3 $6006 \mathrm{U}$ processor.

A proposed network architecture would use loT devices to turn the hospital into an loT-enabled unit. A proposal has been made to use COVID-19 to more rapidly detect and isolate COVID-19 subjects. The loTenabled alarm system is prototyped using a Raspberry $\mathrm{Pi}$-based loT device. The Raspberry $\mathrm{Pi}$ device is virtually connected to the server, which enables the server to utilise COVID-19 positive subjects for communicating with the loT network, thereby allowing for various actions on the network. Classified images sent to the Raspberry $\mathrm{Pi}$ are used to programme and activate results, like turning on a red LED and a buzzer when COVID-19 is detected and activating a blue LED when "Normal" is detected. This is an incredibly simple Proof of Concept (POC) demonstrating the broad potential benefits that could result from the application of this new technology. This POC demonstrates that this new technology could help the medical staff save time and assist in saving lives by improving early and accurate detection.

\section{RESULTS}

The different performance evaluation criteria were used to validate our mathematical model based on pathological data and body vitals and the evaluation parameters have been given in Table $\mathbf{3}$.

From Table 3, the authors have found that the two major deciding factors in the model selection are sensitivity and specificity. "COVID" (target class) was detected as "COVID" (output class). This implies that "COVID" (target class) is sensitive. The sensitivity test for "COVID" returns a higher accuracy. The authors may notice that the model was made to be equivalent to the true positives and false negatives count. The authors have used the concept of transfer learning for the application of the Al-based radiography-assisted technique. It's important when training the model with non-matching output classes. It helps to reduce training time, without requiring the model to be developed from scratch.

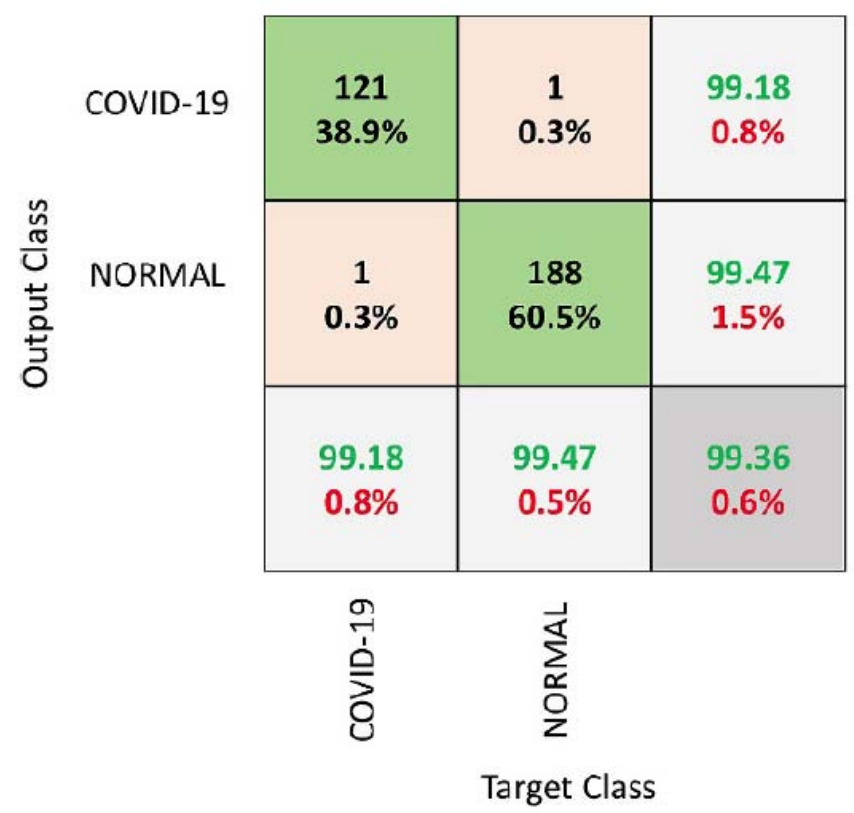

Figure 7: Confusion matrix for the suggested ensemble DL architecture on the test. 

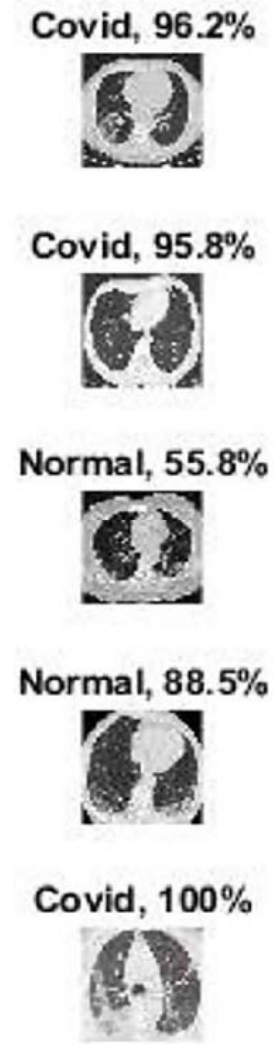
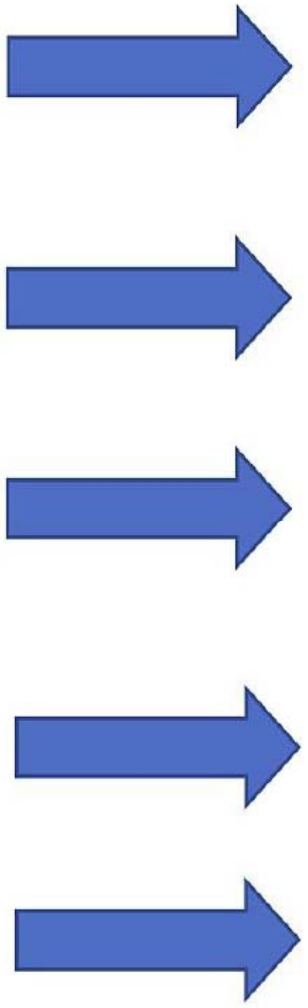

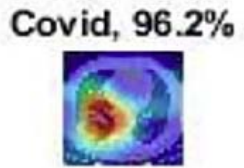

Covid, $95.8 \%$
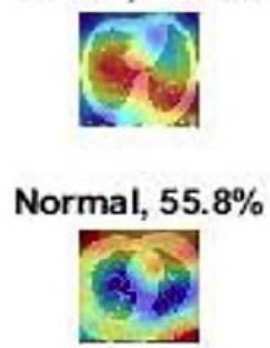

Normal, $88.5 \%$

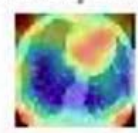

Covid, $100 \%$

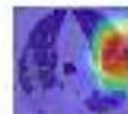

Figure 8: Classification Activation Mapping (CAM): Annotated Results to Show Affected Lung Area.

As shown in Figure 7, the developed EL was evaluated on the test data. The overall accuracy for the COVID-19 class is 99.36 percent. The proposed architecture was shown to have a precision of 99.18 percent, specificity of 99.47 percent, sensitivity or recall of 99.18 percent, and $\mathrm{F}-1$ score of 0.9918 . The model's performance is further validated using a visualisation technique called Class Activation Mapping (CAM) [54]. This method enables us to visualise the reason for classification using a gradient map or score. In our case, the red-colored zones allowed the model to classify the image as belonging to any class. The following Figure 8 illustrates some sample observations on images along with the CAM zones.

\section{DISCUSSION}

The validation analysis for the proposed model is described in Figure 9. The results indicate that the proposed framework outperforms existing deep learning models. In terms of sensitivity, the area under the curve (AUC), specificity, F-measure, and accuracy the proposed framework outperforms existing models.

FRCR has demonstrated a 98 percent accuracy rate on CXR images. DL-CRC has a classification accuracy of was 93.94 percent. 3DCNN had a sensitivity of 86.9 percent, a specificity of 90.1 percent, and an accuracy of 87.5 percent, respectively. CheXNet achieved 97.9 percent classification accuracy, 97.9 percent specificity, and 98.8 percent sensitivity, respectively. Another architecture VGG19 demonstrated a total sensitivity of $93 \%$. CovidGAN achieved accuracy of $95 \%$. ACCA's classification accuracy was 91.1 percent. The classification accuracy of AD3D-MIL was 97.9 percent, the AUC was 99 percent, and the kappa score was 95.7 percent. As a result, the developed framework outperforms these techniques with a 99.36 percent accuracy and a 99.18 percent F-measure. Additionally, the proposed model achieves 99.18, 99.47, and 99.21 for sensitivity, specificity, and AUC, respectively. Thus, when compared with existing coronavirus diagnosis systems, the suggested model has a higher degree of reliability in terms of performance.

Our current setup uses Intel $\circledast$ Neural Compute Stick and Intel ${ }^{\circledR}$ Al Dev cloud. The cloud is vital in any loT ecosystem implementation. We focus on two key goals: (i) Isolating COVID patients at the point of care, and (ii) Hospitalization. The current setup uses Intel ${ }^{8}$ Al dev cloud, a free cloud. No other free cloud can compare to this one's benefits. 


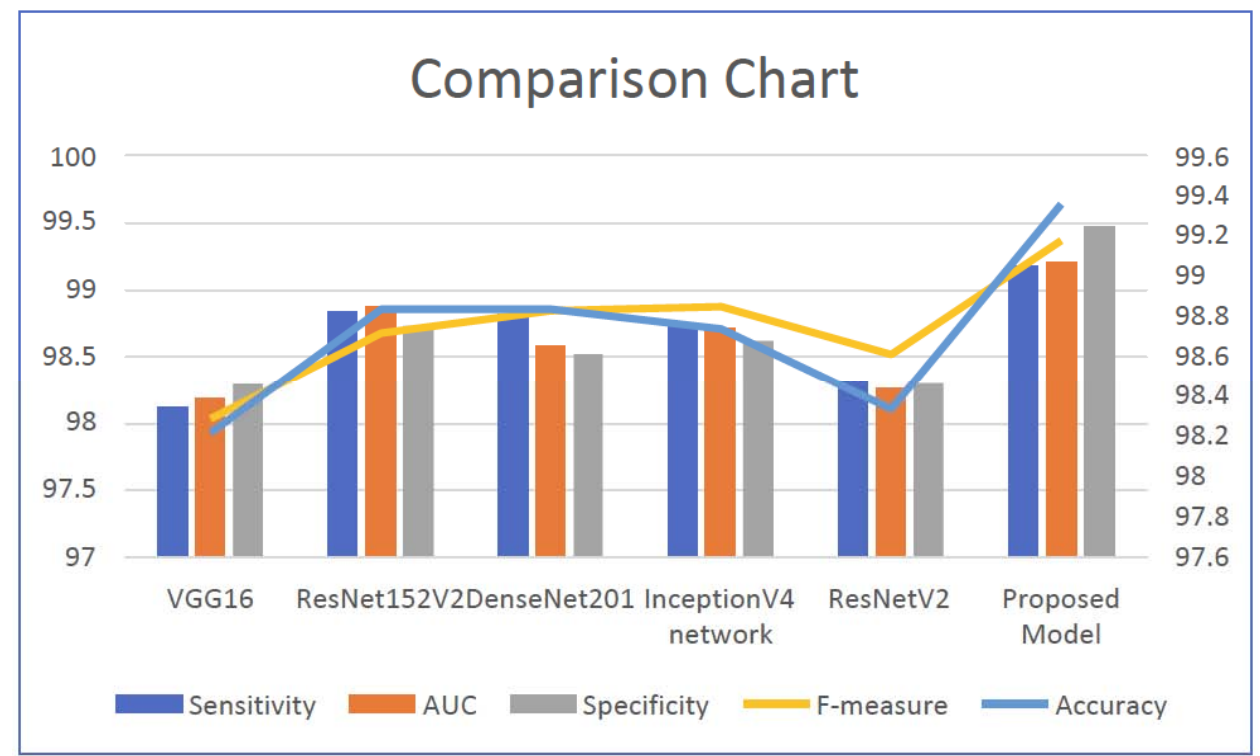

Figure 9: The performance of the proposed ensemble technique is compared to comparable deep learning models.

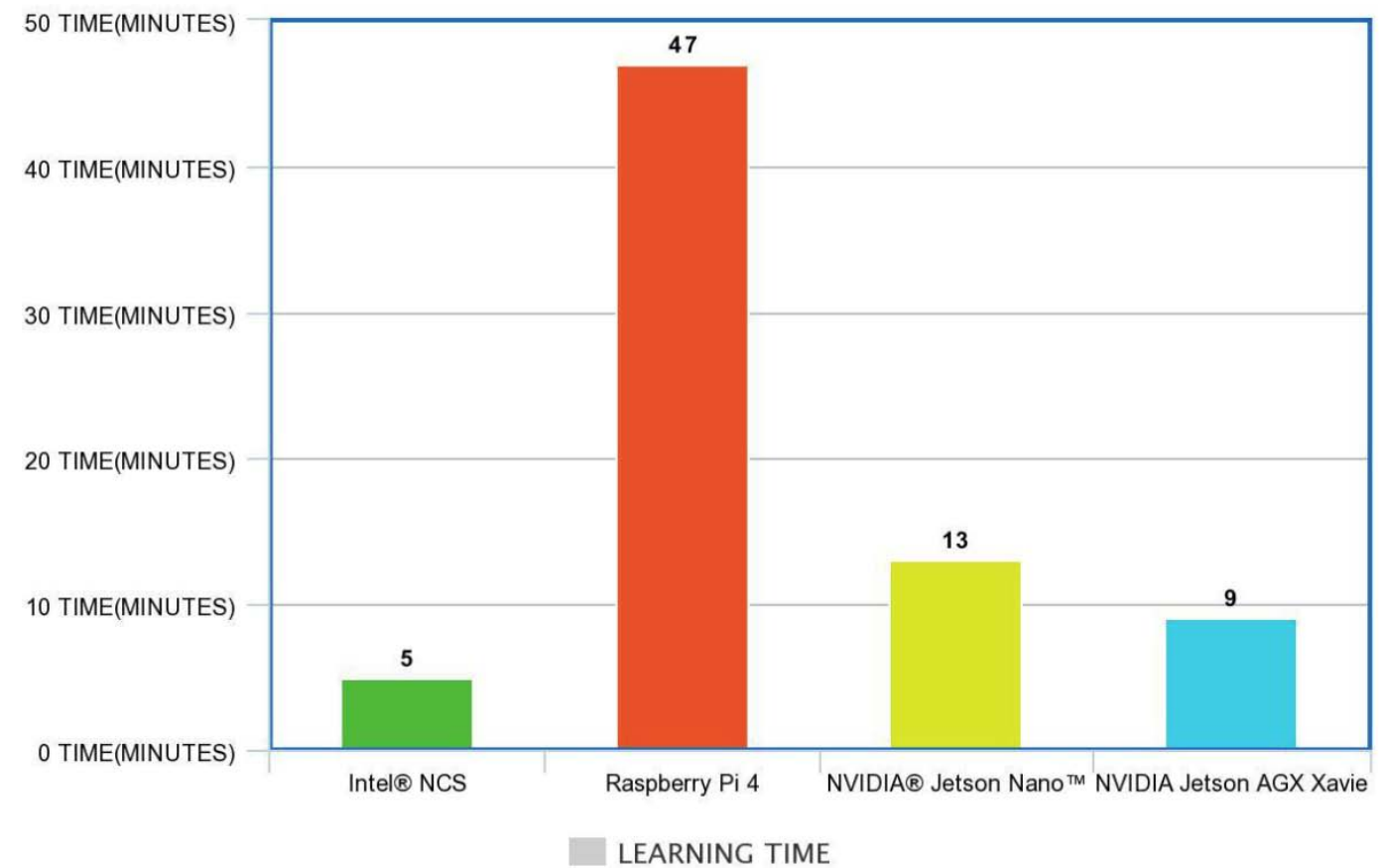

Figure 10: The comparison of learning time of different SBCs.

The Intel $\circledast$ neural Compute stick platform provides lower computational and time complexity when compared to the Raspberry pi 4 GB module, NVIDIA Jetson Nano, and NVIDIA Xavier CUDA. Figures 10 and 11 show the learning and prediction time complexity in various Single-Board Computers.

\section{CONCLUSION}

The article outlines a method for real-time diagnosis of suspected COVID-19 cases including a three-step screening process. Using ensemble deep learning, a fully automated loT-based coronavirus diagnosis system was designed. This cutting-edge system is now running on an Intel-based computer platform and making use of Intel's Al development cloud (free). A stand-alone application including only the models can be integrated with enterprise systems such as hospital information systems, radiology information systems, and laboratory information systems for diagnostic centers or point of care facilities as part of the future scope. Four deep learning models were combined in 


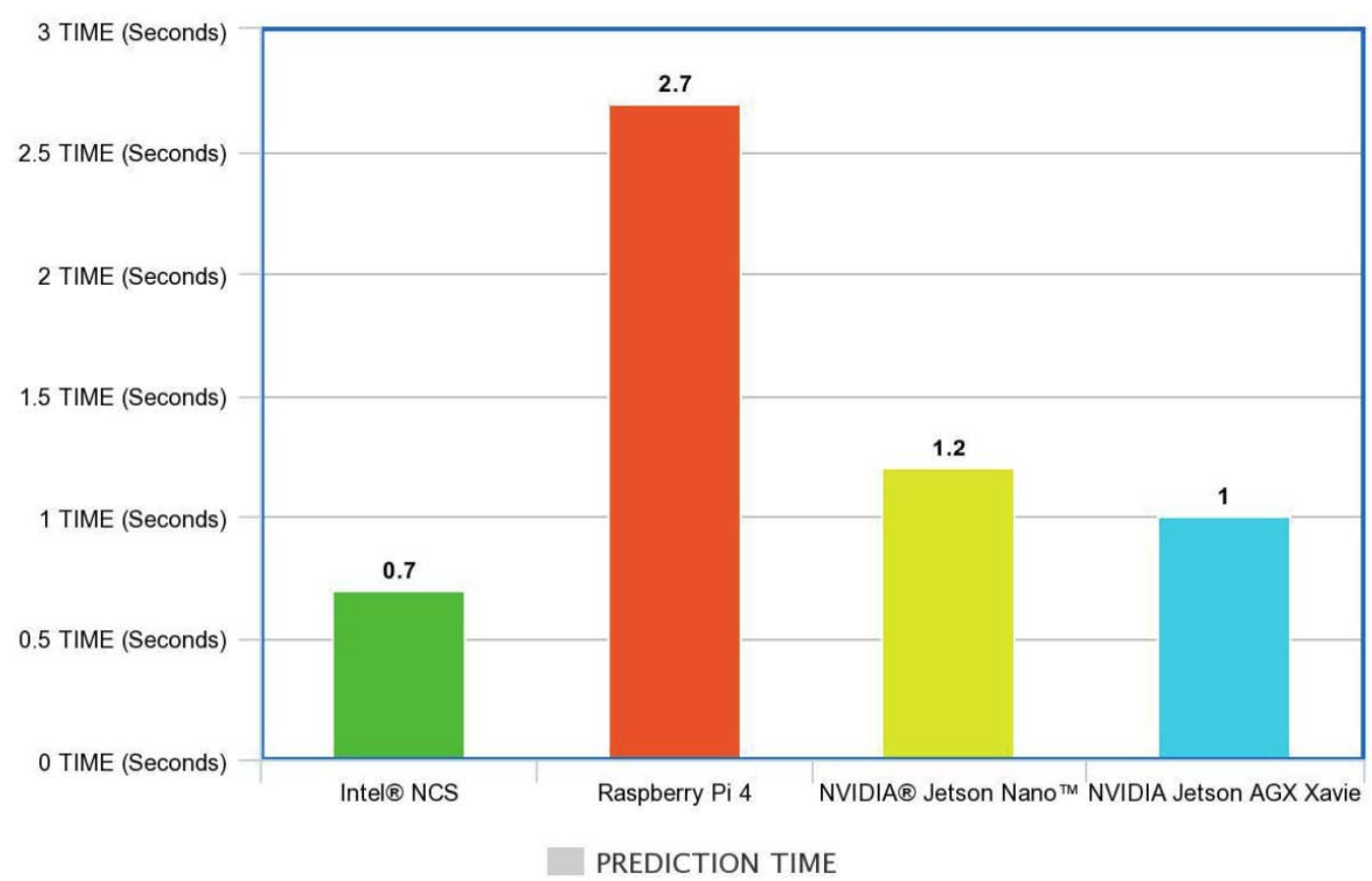

Figure 11: The comparison of prediction time of different SBCs.

the proposed framework, including DenseNet201, InceptionResNetV2, ResNet152V2, and VGG16. The proposed model's performance was evaluated using binary-class HRCT datasets. The proposed framework outperforms existing models by 1.2832 percent, 1.1837 percent, 1.3281 percent, 1.3928 percent, and 1.2837 percent, respectively, in terms of F-score, accuracy, specificity, sensitivity, and AUC. This proposed algorithm will be developed into software, which will then aid health practitioners and other medical staff, and thus contribute to the diagnostic process. Comparative analysis has demonstrated that the proposed architecture can assist medical professionals in diagnosing COVID-19 suspected patients efficiently and timely. This model is meant to supplement the RTPCR Process, rather than take a place in it. Additionally, it is a highly pocket-friendly solution for the prediction of coronavirus-positive cases. The use of a chain of secondary hypotheses to diminish the false negatives is done. With this methodology, we hope to speed up the testing process, and as a result, increase the number of tests conducted per day. Additionally, it may also help in institutions without RT- PCR facilities.

\section{ACKNOWLEDGEMENTS}

The authors also acknowledge The Neotia University and Adamas University for providing excellent research infrastructure and for necessary funding.

\section{DECLARATIONS}

\section{Funding}

Provided by The Neotia University and Adamas University, Kolkata, India.

\section{Conflicts of Interest/Competing Interests}

Not applicable.

\section{Availability of Data and Material}

Not applicable.

\section{Code Availability}

Not applicable.

\section{Authors' Contributions}

Mr. Swarnava Biswas and Mr. Debajit Sen contributed equally to this research paper.

\section{Ethics Approval}

Not applicable.

\section{Consent to Participate}

Not applicable.

\section{Consent for Publication}

Not applicable. 


\section{REFERENCES}

[1] Akan OB, Andreev S, Dobre C. Internet of things and sensor Networks. IEEE Communications Magazine 2019; 57(2): 40. https://doi.org/10.1109/MCOM.2019.8647109

[2] Runkler TA. Data Visualization. Data Analytics. Springer; 2020; pp. 37-59. https://doi.org/10.1007/978-3-658-29779-4 4

[3] Ebadi A, Xi P, Tremblay S, Spencer B, Pall R, Wong A. Understanding the temporal evolution of COVID-19 research through machine learning and natural language processing. Scientometrics 2021; 126(1): 725-39. https://doi.org/10.1007/s11192-020-03744-7

[4] Gubala V, Harris LF, Ricco AJ, Tan MX, Williams DE. Point of care diagnostics: status and future. Analytical chemistry 2012; 84(2): 487-515. https://doi.org/10.1021/ac2030199

[5] Yuehong $Y$, Zeng $Y$, Chen $X$, Fan $Y$. The internet of things in healthcare: An overview. Journal of Industrial Information Integration 2016; 1: 3-13. https://doi.org/10.1016/j.jii.2016.03.004

[6] Farahani B, Firouzi F, Chakrabarty K. Healthcare iot. Intelligent Internet of Things. Springer 2020; pp. 515-45. https://doi.org/10.1007/978-3-030-30367-9 11

[7] Chakraborty I, Maity P. COVID-19 outbreak: Migration, effects on society, global environment and prevention. Science of the Total Environment 2020; 728: 138882. https://doi.org/10.1016/j.scitotenv.2020.138882

[8] Afzal A. Molecular diagnostic technologies for COVID-19: Limitations and challenges. Journal of Advanced Research 2020. https://doi.org/10.1016/j.jare.2020.08.002

[9] Horry MJ, Chakraborty S, Paul M, Ulhaq A, Pradhan B, Saha $\mathrm{M}$, et al. COVID-19 detection through transfer learning using multimodal imaging data. IEEE Access 2020; 8: 149808-24. https://doi.org/10.1109/ACCESS.2020.3016780

[10] Ho TKK, Gwak J, Prakash O, Song J-I, Park CM. Utilizing pretrained deep learning models for automated pulmonary tuberculosis detection using chest radiography. Asian conference on intelligent information and database systems: Springer 2019; pp. 395-403.

https://doi.org/10.1007/978-3-030-14802-7_34

[11] Narin A, Kaya C, Pamuk Z. Automatic detection of coronavirus disease (covid-19) using X-ray images and deep convolutional neural networks. Pattern Analysis and Applications 2021; 1-14. https://doi.org/10.1007/s10044-021-00984-y

[12] Abbas A, Abdelsamea MM, Gaber MM. Classification of COVID-19 in chest X-ray images using DeTraC deep convolutional neural network. Applied Intelligence 2021; 51(2): 854-64.

https://doi.org/10.1007/s10489-020-01829-7

[13] Ahmed I, Ahmad A, Jeon G. An iot based deep learning framework for early assessment of covid-19. IEEE Internet of Things Journal 2020. https://doi.org/10.1109/JIOT.2020.3034074

[14] Chowdhury ME, Rahman T, Khandakar A, Mazhar R, Kadir MA, Mahbub ZB, et al. Can Al help in screening viral and COVID-19 pneumonia? IEEE Access 2020; 8: 132665-76. https://doi.org/10.1109/ACCESS.2020.3010287

[15] Han Z, Wei B, Hong Y, Li T, Cong J, Zhu X, et al. Accurate screening of COVID-19 using attention- based deep 3D multiple instance learning. IEEE Transactions on Medical Imaging 2020; 39(8): 2584-94. https://doi.org/10.1109/TMI.2020.2996256

[16] Qian X, Fu H, Shi W, Chen T, Fu Y, Shan F, et al. M \$^ 3\$ Lung-Sys: A Deep Learning System for Multi-Class Lung Pneumonia Screening From CT Imaging. IEEE Journal of Biomedical and Health Informatics 2020; 24(12): 3539-50. https://doi.org/10.1109/JBHI.2020.3030853
Zhang J, Xie Y, Pang G, Liao Z, Verjans J, Li W, et al. Viral pneumonia screening on chest $X$-ray images using confidence-aware anomaly detection. arXiv preprint arXiv: 2003123382020 .

[18] Wang L, Lin ZQ, Wong A. Covid-net: A tailored deep convolutional neural network design for detection of covid-19 cases from chest x-ray images. Scientific Reports 2020; 10(1): 1-12. https://doi.org/10.1038/s41598-020-76550-z

[19] Makris A, Kontopoulos I, Tserpes K. COVID-19 detection from chest X-Ray images using Deep Learning and Convolutional Neural Networks. 11th Hellenic Conference on Artificial Intelligence 2020; pp. 60-6. https://doi.org/10.1145/3411408.3411416

[20] Sakib S, Tazrin T, Fouda MM, Fadlullah ZM, Guizani M. DLCRC: deep learning-based chest radiograph classification for COVID-19 detection: a novel approach. IEEE Access 2020; 8: $171575-89$ https://doi.org/10.1109/ACCESS.2020.3025010

[21] Farooq M, Hafeez A. Covid-resnet: A deep learning framework for screening of covid19 from radiographs. arXiv preprint arXiv: 2003143952020.

[22] Waheed A, Goyal M, Gupta D, Khanna A, Al-Turjman F, Pinheiro PR. Covidgan: data augmentation using auxiliary classifier gan for improved covid-19 detection. IEEE Access 2020; 8: 91916-23 https://doi.org/10.1109/ACCESS.2020.2994762

[23] Gianchandani N, Jaiswal A, Singh D, Kumar V, Kaur M. Rapid COVID-19 diagnosis using ensemble deep transfer learning models from chest radiographic images. Journal of Ambient Intelligence and Humanized Computing 2020; 1-13. https://doi.org/10.1007/s12652-020-02669-6

[24] Singh D, Kumar V, Yadav V, Kaur M. Deep neural networkbased screening model for COVID-19- infected patients using chest X-ray images. International Journal of Pattern Recognition and Artificial Intelligence 2021; 35(03): 2151004. https://doi.org/10.1142/S0218001421510046

[25] Singh D, Kumar V, Kaur M. Densely connected convolutional networks-based COVID-19 screening model. Applied Intelligence 2021; 51(5): 3044-51. https://doi.org/10.1007/s10489-020-02149-6

[26] Apostolopoulos ID, Mpesiana TA. Covid-19: automatic detection from $\mathrm{x}$-ray images utilizing transfer learning with convolutional neural networks. Physical and Engineering Sciences in Medicine 2020; 43(2): 635-40 https://doi.org/10.1007/s13246-020-00865-4

[27] Basavegowda HS, Dagnew G. Deep learning approach for microarray cancer data classification. CAAl Trans Intell Technol 2020; 5(1): 22-33.

https://doi.org/10.1049/trit.2019.0028

[28] Ghosh S, Shivakumara P, Roy P, Pal U, Lu T. Graphology based handwritten character analysis for human behaviour identification. CAAI Trans Intell Technol 2020; 5(1): 55-65. https://doi.org/10.1049/trit.2019.0051

[29] Gupta B, Tiwari M, Lamba SS. Visibility improvement and mass segmentation of mammogram images using quantile separated histogram equalisation with local contrast enhancement. CAAI Transactions on Intelligence Technology 2019; 4(2): 73-9. https://doi.org/10.1049/trit.2018.1006

[30] Brihn A, Chang J, OYong K, Balter S, Terashita D, Rubin Z, et al. Diagnostic Performance of an Antigen Test with RTPCR for the Detection of SARS-CoV-2 in a Hospital SettingLos Angeles County, California, June-August 2020. Morbidity and Mortality Weekly Report 2021; 70(19): 702. https://doi.org/10.15585/mmwr.mm7019a3

[31] Shakouri S, Bakhshali MA, Layegh P, Kiani B, Masoumi F, Nakhaei SA, et al. COVID19-CT-dataset: an open-access 
chest CT image repository of $1000+$ patients with confirmed COVID-19 diagnosis. BMC Research Notes 2021; 14(1): 1-3. https://doi.org/10.1186/s13104-021-05592-x

[32] Loussaief $S$, Abdelkrim A. Deep learning vs. bag of features in machine learning for image classification 2018 International Conference on Advanced Systems and Electric Technologies (IC ASET): IEEE 2018; pp. 6-10. https://doi.org/10.1109/ASET.2018.8379825

[33] Schapire RE. Explaining adaboost. Empirical inference. Springer 2013; pp. 37-52. https://doi.org/10.1007/978-3-642-41136-6 5

[34] Seiffert C, Khoshgoftaar TM, Van Hulse J, Napolitano A. RUSBoost: Improving classification performance when training data is skewed 2008 19th International Conference on Pattern Recognition: IEEE 2008; pp. 1-4. https://doi.org/10.1109/ICPR.2008.4761297

[35] Guan W-J, Ni Z-Y, Hu Y, Liang W-H, Ou C-Q, He J-X, et al. Clinical characteristics of coronavirus disease 2019 in China. New England Journal of Medicine 2020; 382(18): 1708-20. https://doi.org/10.1056/NEJMoa2002032

[36] Chen N, Zhou M, Dong X, Qu J, Gong F, Han Y, et al. Epidemiological and clinical characteristics of 99 cases of 2019 novel coronavirus pneumonia in Wuhan, China: a descriptive study. The Lancet 2020; 395(10223): 507-13. https://doi.org/10.1016/S0140-6736(20)30211-7

[37] Simpson S, Kay FU, Abbara S, Bhalla S, Chung JH, Chung $\mathrm{M}$, et al. Radiological society of north America expert consensus document on reporting chest CT findings related to COVID-19: endorsed by the society of thoracic Radiology, the American college of Radiology, and RSNA. Radiology: Cardiothoracic Imaging 2020; 2(2): e200152. https://doi.org/10.1148/ryct.2020200152

[38] Ritschard G. Computing and using the deviance with classification trees. COMPSTAT 2006- Proceedings in Computational Statistics. Springer 2006; pp. 55-66. https://doi.org/10.1007/978-3-7908-1709-6 5

[39] Kayri M, Kayri I. The comparison of Gini and Twoing algorithms in terms of predictive ability and misclassification cost in data mining: an empirical study. Databases 2015; 3 : 5 .

https://doi.org/10.14445/22312803/lJCTT-V27P105

[40] Baczkowski A, Joanes D, Shamia G. The distribution of a generalized diversity index due to Good. Environmental and Ecological Statistics 2000; 7(4): 329-42. https://doi.org/10.1023/A:1026567414861

[41] Shih K-H, Chiu C-T, Lin J-A, Bu Y-Y. Real-time object detection with reduced region proposal network via multifeature concatenation. IEEE Transactions on Neural Networks and Learning Systems 2019; 31(6): 2164-73. https://doi.org/10.1109/TNNLS.2019.2929059

[42] Zhou Y, Li G, Li H. Automatic cataract classification using deep neural network with discrete state transition. IEEE Transactions on Medical Imaging 2019; 39(2): 436-46. https://doi.org/10.1109/TMI.2019.2928229

[43] Yang W, Cao Q, Qin L, Wang X, Cheng Z, Pan A, et al. Clinical characteristics and imaging manifestations of the
2019 novel coronavirus disease (COVID-19): a multi-center study in Wenzhou city, Zhejiang, China. Journal of Infection 2020; 80(4): 388-93 https://doi.org/10.1016/j.jinf.2020.02.016

[44] Yoon SH, Lee KH, Kim JY, Lee YK, Ko H, Kim KH, et al. Chest radiographic and $\mathrm{CT}$ findings of the 2019 novel coronavirus disease (COVID-19): analysis of nine patients treated in Korea. Korean Journal of Radiology 2020; 21(4): 494.

https://doi.org/10.3348/kjr.2020.0132

[45] Rodrigues J, Hare S, Edey A, Devaraj A, Jacob J, Johnstone $A$, et al. An update on COVID-19 for the radiologist-A British society of Thoracic Imaging statement. Clinical Radiology 2020; 75(5): 323-5. https://doi.org/10.1016/i.crad.2020.03.003

[46] Ludvigsson JF. Systematic review of COVID-19 in children shows milder cases and a better prognosis than adults. Acta Paediatrica 2020; 109(6): 1088-95. https://doi.org/10.1111/apa.15270

[47] Holshue ML, DeBolt C, Lindquist S, Lofy KH, Wiesman J, Bruce $\mathrm{H}$, et al. First case of 2019 novel coronavirus in the United States. New England Journal of Medicine 2020. https://doi.org/10.1056/NEJMoa2001191

[48] Yang R, Li X, Liu H, Zhen Y, Zhang X, Xiong Q, et al. Chest CT severity score: an imaging tool for assessing severe COVID-19. Radiology: Cardiothoracic Imaging 2020; 2(2): e200047. https://doi.org/10.1148/ryct.2020200047

[49] Zhang W, Thurow K, Stoll R. A knowledge-based telemonitoring platform for application in remote healthcare. International Journal of Computers Communications \& Control 2014; 9(5): 644-54. https://doi.org/10.15837/ijccc.2014.5.661

[50] Dong J, Zhuang D, Huang Y, Fu J. Advances in multi-sensor data fusion: Algorithms and applications. Sensors 2009; 9(10): 7771-84 https://doi.org/10.3390/s91007771

[51] Gevaert CM, García-Haro FJ. A comparison of STARFM and an unmixing-based algorithm for Landsat and MODIS data fusion. Remote Sensing of Environment 2015; 156: 34-44. https://doi.org/10.1016/j.rse.2014.09.012

[52] Fourati $H$. Heterogeneous data fusion algorithm for pedestrian navigation via foot-mounted inertial measurement unit and complementary filter. IEEE Transactions on Instrumentation and Measurement 2014; 64(1): 221-9 https://doi.org/10.1109/TIM.2014.2335912

[53] Ambühl L, Menendez M. Data fusion algorithm for macroscopic fundamental diagram estimation. Transportation Research Part C: Emerging Technologies 2016; 71: 184-97. https://doi.org/10.1016/j.trc.2016.07.013

[54] Selvaraju RR, Cogswell M, Das A, Vedantam R, Parikh D, Batra D. Grad-cam: Visual explanations from deep networks via gradient-based localization. Proceedings of the IEEE international conference on computer vision 2017; pp. 61826 .

https://doi.org/10.1109/ICCV.2017.74

\footnotetext{
Received on 18-09-2021

https://doi.org/10.6000/1929-6029.2021.10.14
}

Accepted on 22-10-2021

Published on 04-11-2021

(C) 2021 Biswas et al.; Licensee Lifescience Global.

This is an open access article licensed under the terms of the Creative Commons Attribution License (http://creativecommons.org/licenses/by/4.0/) which permits unrestricted use, distribution and reproduction in any medium, provided the work is properly cited. 